Meta

Journal des traducteurs

Translators' Journal

\title{
Je, tu, il ou elle, nous, vous, ils ou elles
}

Daniel L. Bon

Volume 29, numéro 2, juin 1984

URI : https://id.erudit.org/iderudit/002100ar

DOI : https://doi.org/10.7202/002100ar

Aller au sommaire du numéro

Éditeur(s)

Les Presses de l'Université de Montréal

ISSN

0026-0452 (imprimé)

1492-1421 (numérique)

Découvrir la revue

Citer cette note

Bon, D. L. (1984). Je, tu, il ou elle, nous, vous, ils ou elles. Meta, 29(2), 218-220.

https://doi.org/10.7202/002100ar d'utilisation que vous pouvez consulter en ligne.

https://apropos.erudit.org/fr/usagers/politique-dutilisation/ 


\section{JE, TU, IL OU ELLE \\ NOUS, VOUS, ILS OU ELLES}

La bataille du he or she, ou si l'on préfere l'offensive du chairperson, fait rage dans les rédactions des publications de langue anglaise. Elle oppose par ailleurs mari et femme, frère et soeur, quand ce n'est pas mère et fille, et a coûté maintes amitiés parmi les protagonistes d'une nouvelle et contemporaine querelle des Anciens et des Modernes. Elle perturbe même à ce point les esprits que jaillissent de plumes d'ordinaire aussi sereines que sûres et éclairées d'étonnantes combinaisons que l'on ne sait au juste à quoi attribuer, mais que condamnent avec la même fermeté les Messieurs Thomas ${ }^{1}$ et Colin ${ }^{2}$ de l'idiome anglo-saxon, Henry W. Fowler ${ }^{3}$ et Elwyn B. White ${ }^{4}$. C'est ainsi que, aveuglement ou épouvante, de plus en plus d'anglophones cèdent à un instinct qui les porte à tordre le cou au bon usage pour dire et écrire : "Everyone I met there thought they were owed a living" et "It was the sort of place where anyone with something to say always thought they should say it." Ces constructions sont, bien entendu, aussi peu correctes qu'illogiques.

La querelle en question ne devrait en principe concerner ni les francophones ni le français, tant il est vrai que chaque langue a son génie, qu'il est bien rare que les problèmes de l'une soient les problèmes des autres, et encore plus que les solutions admissibles dans un cas le soient dans les autres. Néanmoins, au Canada, il se trouve des individus - surtout, mais pas uniquement chez les activistes féministes - pour vouloir universaliser les "solutions" façonnées par leurs homologues anglophones. He or she devient " il ou elle " et Ms.; "Madelle", tandis que le débat autour de chairperson, qui se règle à l'amiable dès que

1. Adolphe V. Thomas, auteur du Dictionnaire des difficultés de la langue française (Larousse).

2. Jean-Paul Colin, auteur du Nouveau Dictionnaire des difficultés du français (Hachette-Tchou)

3. Auteur de Modern English Usage (Oxford University Press).

4. Coauteur de The Elements of Style (Macmillan) 
l'identité de la personne qui doit présider est connue, tend à dégénérer tant que plane l'incertitude.

Au risque de passer pour sexiste et de se faire traiter de phallocrate, il faut rappeler à celles et à ceux qui voudraient greffer sur le français des habitudes et modèles d'évolution sémantique et linguistique créés par l'anglais : d'abord, que l'excès, en tout, reste un défaut ; ensuite, que si dans certains cas telle ou telle innovation peut passer, à trop vouloir étendre et retailler le français pour satisfaire aux normes du moment (quelles que puissent être par ailleurs les intentions de ceux qui en sentent le besoin) on finit par le coucher dans un lit de Procuste dont il ne peut se relever que mal en point. Surtout là où, comme en Amérique, il a déjà dû partager dans des conditions si défavorables et pendant si longtemps la couche de l'anglais. Diantre! Est-ce précisément au moment où disparaissent les derniers " chars usés " qui fonctionnaient au " gaz " que la langue française devrait se retrancher derrière des ill(s) ou elle(s) probablement aussi inutiles que lourds, simplement parce que l'anglais en a décrété ainsi en ce qui le concerne (et au risque de commettre des horreurs grammaticales)? Non, non, non! Trois fois non! Le français n'est pas l'anglais et ne saurait le devenir s'il veut perdurer. Les calques grammaticaux sont encore plus pernicieux que les emprunts de vocables que rien ne justifie, puisque si les seconds sont immédiatement perceptibles, les premiers peuvent parfaitement se tapir derrière une façade de mots irréprochablement français.

Cela ne nous condamne pas pour autant, et de beaucoup s'en faut, à reléguer les femmes à un très secondaire arrière-plan. Car le français, on l'oublie trop souvent dans le feu de l'action passionnée que mènent des militantes féminines en majorité anglophones, n'est pas du tout dépourvu des moyens nécessaires à la mise au point d'une solution. Pour commencer, notons que, contrairement à l'anglais qui, au niveau de l'article, n'exprime pas le genre ( $a$ dancer peut tout aussi bien être une danseuse qu'un danseur), le français, lui, marque toujours un genre - qui ne correspond du reste pas nécessairement au sexe de l'individu dont on parle. Quand une estafette, appelée par une recrue qu'elle doit accompagner à l'escadron d'accueil et d'instruction, il y a tout lieu de supposer, nonobstant les articles, que chacune des personnes visées est de sexe masculin. Cela peut servir à mettre en relief le peu d'intérêt qu'il y a au fond à se préoccuper des problèmes que se crée l'anglais avec chairperson (voire, un de ces jours, postperson).

D'aucuns voudront faire valoir que ce qui précède ne consitue en fait qu'une maigre exception à ce qui reste un partage éminemment léonin de la réalité des hommes, des femmes et de leurs métiers en français. C'est un fait. Il faut en convenir et le concéder volontiers. Mais (car il y a un mais...) pour peu que l'on veuille bien lui en donner l'occasion, précisément en raison de la nécessité à laquelle il ne peut échapper de fixer un genre, le français est en mesure de faire jouer des mécanismes dont ne dispose pas l'anglais pour aplanir le genre de difficultés dont il est ici question. Une certaine rigidité, qui contribue du reste dans une large mesure à la clarté que l'on reconnaît généralement au français, ne va pas sans s'accompagner d'une certaine souplesse qui pourrait faire beaucoup pour la paix des ménages. À quoi bon, par conséquent aller s'empêtrer dans des formulations qui ne sauraient manquer d'être abracadabrantes si elles s'obstinaient à rendre servilement à la femme de César, en même temps qu'à César, ce qui appartient à César, dans l'adaptation de phrases comme celle-ci : "It is the right of every Canadian, should he or she choose to avail himself or herself of it. to... " (On remarquera au passage que malgré toute sa complication la phrase anglaise que l'on vient de lire ne règle absolument rien au fond, puisque la préséance qu'elle accorde au pronom masculin est au moins aussi contestable que l'emploi de ce seul pronom pourrait l'être.)

Jouons de l'article et des terminaisons féminisantes. Même si l'anglais tient absolument à créer postperson, restons-en, de grâce, à "factrice "5. Généralisons "avocate", "doctoresse", "chirurgienne". Osons une pilote, une ingénieur, une ministre (à défaut de une "ministresse ", comme l'écrit parfois plaisamment le Canard enchaînê). Et n'oublions pas que, bien souvent, ce ne sont pas des hommes, mais des femmes, qui ralentissent l'évolution en ce sens. Ainsi, il est des présidentes d'assemblées, législatives ou autres, qui préfêrent se faire donner du " président ". Elles voient en effet condescendance et risque de méprise dans la forme féminine du terme, parce qu'il fut un temps où, les choses étant ce qu'elles étaient, seul un homme pouvait présider aux destinées de quoi que ce soit (hormis, on imagine, un cercle féminin). Une présidente ne pouvait donc être autre chose que l'épouse d'un président, par définition, comme une générale était celle d'un général et une ambassadrice celle d'un ambassadeur (et ainsi de suite). Il conviendrait de faire valoir auprès de ces dames que ces temps-là sont révolus. Les convaincre que l'humanité francophone leur sera tout aussi éternellement reconnaissante de faire en sorte que puisse être entérinée l'évolution de ces dernières décennies que de bien vouloir s'abstenir de s'affubler de tristes cravates ou de tailleurs gris banquier. Et encore, puisqu'elles n'ont pas l'air d'en être persuadées, qu'il leur incombe d'autant plus qu'elles exercent des fonctions éminentes de souligner en tout et partout et leur féminité et leur égalité. Lorsque, articles et terminaisons aidant, la condition féminine aura été valorisée au point que petites filles et petits garçons n'auront plus l'impression que les plus hautes fonctions de la carrière diplomatique sont réservées aux hommes, peut-être n'y aura-t-il plus de raison de voir, dans l'emploi du pronom masculin (voire du terme " homme " lui-même), pour, en francais, marquer le neutre, une discrimination à l'endroit des femmes. Qui sait si alors ce ne seront pas les

5. Maurice Grevisse, le Bon usage, Duculot, 8૯ éd. re vue, 247 , rem. 5, p. 185 . 
hommes qui s'offusqueront de cet emploi du masculin dans des fonctions neutres...

Cette dernière remarque en appelle une autre, qui servira de conclusion et, peut-être, à mettre en lumière le caractère artificiel de l'extension de la querelle de he or she au français. Chez les messieurs, une réaction comme celle qui vient d'être évoquée serait bien entendu ridicule. Elle le serait davantage encore, cependant, si elle était provoquée, parmi les francophones, par quelque "révolte" de leurs cousins anglophones contre, par exemple, ce qu'ils voudraient voir d'antimasculin dans une certaine spécialisation des pronoms de la troisième personne que la tradition perpétue en anglais. En effet, dès lors que quelque chose ou quelque animal se trouve investi d'attributs rassurants, agréables, réconfortants, voire maternels, tel le navire qui vous ramènera à bon port ou l'habituel train de cinq heures, on en fait un she "There she goes». Par contre, sitôt qu'il s'agit d'un être ou d'un objet suspect, sombre, velu, menaçant, on y voit un he : "Where is he now?", dit-on de l'araignée dont on redoute la réapparition. Sachons ne pas trop confondre le fond et la forme. Le français comme l'anglais ont évolué dans des contextes dominés par les hommes et conservent la marque de cette évolution. L'un comme l'autre peuvent, et doivent, progressivement se débarrasser de cette marque. Cela ne veut pas dire qu'ils peuvent et doivent le faire simultanément et de la même façon. Cela ne veut pas davantage dire qu'il ne faut en aucun cas laisser subsister la moindre trace de l'" ancien régime » (ne serait-ce que pour mémoire) ou qu'il faille extirper de la langue jusqu'à la dernière caractérisation affective que peut traduire l'emploi d'un genre plutôt que d'un autre. Trop, c'est trop...

DANIEL L. BON 